\title{
Endodontic Management of Mesotaurodontic Tooth with Endocrown: A Case Report
}

\author{
1James D Raj, ${ }^{2}$ Niveditha MS Babu, ${ }^{3}$ Victor Samuel A
}

\begin{abstract}
Taurodontism is a developmental disturbance of a tooth that lacks constriction at the level of the cementoenamel junction and is characterized by unusually large pulp chambers. This enlarged pulp chamber causes the pulpal floor to be present more apically and bifurcation or trifurcation of the roots. Endodontic therapy of taurodontic tooth always poses a challenging task for clinicians. Moreover, post-endodontic restoration of the tooth with gross destruction should be handled judiciously. This is the first paper which describes the endodontic management of mesotaurodontic tooth with endocrown.
\end{abstract}

Keywords: Endocrown, Mesotaurodont, Taurdontism.

How to cite this article: Raj JD, Babu NMS, Samuel VA. Endodontic Management of Mesotaurodontic Tooth with Endocrown: A Case Report. J Oper Dent Endod 2018;3(2):97-100.

Source of support: Nil

Conflict of interest: None

\section{INTRODUCTION}

Taurodontism is a developmental disturbance of a tooth that lacks constriction at the level of the cementoenamel junction and is characterized by unusually large pulp chambers. This enlarged pulp chamber causes the pulpal floor to be present more apically and bifurcation or trifurcation of the roots. ${ }^{1}$ As a dental anomaly, taurodontism is characterized externally as a bull-like heavily bodied tooth in which crown tends to enlarge at the expense of root. This leads to greater apico-occlusal height and the distance from the bifurcation of roots to the CEJ in taurodont teeth is greater than the occlusocervical distance. ${ }^{2,3}$ The etiology of taurodontism is unclear. The cause can be a failure of Hertwig's epithelial sheath diaphragm to invaginate at the proper horizontal level, resulting in a tooth with short roots, an elongated body, an enlarged

\footnotetext{
${ }^{1}$ Associate Professor, ${ }^{2}$ Senior Lecturer, ${ }^{3}$ Professor

${ }^{1}$ Department of Pedodontics and Preventive Dentistry, SRM Kattankulathur Dental College, SRM Institue of Science and Tehnology, Chennai, Tamil Nadu, India

${ }^{2,3}$ Department of Conservative and Endodontics, Saveetha Dental College, Saveetha University, Chennai, Tamil Nadu, India

Corresponding Author: Victor Samuel A, Professor, Department of Conservative and Endodontics, Saveetha Dental College, Saveetha University, Chennai, Tamil Nadu, India, e-mail: victorsamuel_83@yahoo.co.in
}

pulp, and normal dentine. ${ }^{4,5}$ It may also be present as a variant of the pulp chamber form, which may arise as a result of abnormal dentin development. The latest evidence suggests that there is an association between taurodontism, familial inheritance, genetic malformations, and épithélio-mésenchymateuse induction has also been proposed as a possible etiology. ${ }^{2,6}$

There are subtypes in taurodontism they are hypotaurodontism, mesotaurodontism, and hyper taurodontism based on the relative size of the pulp chamber. ${ }^{7}$ The most prominent taurodontism is prominent amongst the Krapina Neanderthal specimens and the earliest example of taurodontism is that of a Krapina 70000-year-old anthropological specimen. ${ }^{6}$ Any pathology to a taurodontic tooth makes it a challenging task to restore to its form and function. Endodontic management of excessive loss of hard tissues of the crown is a bit more challenging.

Pissis described "mono-block porcelain technique" called the endocrown. ${ }^{8}$ These type of crowns takes support from the pulp chamber and would be anchored to the internal portion and on the cavity margins, thus gaining macromechanical retention provided by the pulpal walls. These crowns are used especially in teeth that are extensively decayed. Usage of endocrown avoids the used of endodontic post as the crown extends almost till the pulpal floor. This paper describes a case report on the endodontic management of taruodontics tooth restored with endocrown.

\section{CASE REPORT}

A 36-year male patient reported to the Department of Conservative Dentistry and Endodontics with pain in relation to lower right back tooth. On clinical examination the lingual part of 47 was grossly destructed with missing distolingual cusp and partial loss of distobuccal cusp due to caries (Fig. 1A) On electric pulp testing (Parkell Edgewood, USA) there was a delayed response at 8 as compared to the contralateral 37 which gave a response at 4 . On thermal (heat) test with gutta percha (GP) stick, there was a lingering pain even after the removal of the stimulus. This narrowed down to the finding as irreversible pulpitis. Radiographic examination revealed the radiolucency approximating the mesial pulp horn of 47 and the tooth appeared to be a taurodont (Fig. 1B) Treatment plan decided was root canal treatment followed 
by endocrown. The treatment plan was explained to the patient and informed consent was obtained.

Root canal therapy was initiated in 47 with proper isolation and irrigation protocol with sodium hypochlorite. (Fig. 2A) Cleaning and shaping were done using HERO shaper $4 \%$ size 20 and $6 \%$ size 25 with adequate irrigation on the first sitting. An intracanal medicament of calcium hydroxide was placed in the canals and closed dressing was given. The patient was asymptomatic after a week; obturation was carried out with matched taper guttapercha cones with AH+ sealer (Dentsply, USA) (Fig. 2B) Tooth preparation was done in 47 to accommodate endocrown. Retraction cord of sizes $\mathrm{OO}$ and two were packed using cord packer (Fig. 3) Two-stage putty wash impression was made using Putty and Light body (Fig. 4) (Betasil Vario, Germany).

The impression was then referred to computer-aided design/computer-aided manufacturing (CAD/CAM) Department for the Optical Impression. After the initial milling, the pre-fired endocrown was tried on the patient to check for the fit and to correct any occlusal disturbance. After which the endocrown was glazed and the tooth was

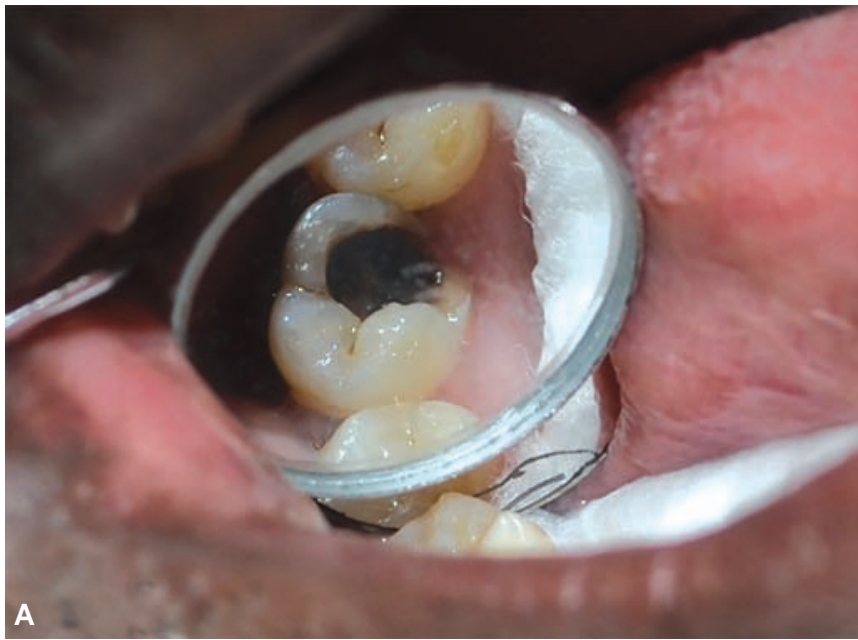

acidly etched for 15 to 20 seconds, rinsed for 20 seconds and air dried (Fig. 5) Bonding agent was applied over the entire surface using applicator tip and light cured for 30 to 40 second. Hydrofluoric acid was used to etch the inner aspect of the endocrown to roughen the surface. Endocrown was luted in 47 using resin cement (RelyX cement) (Fig 6). The patient was called for a regular follow-up. Figure 7 shows 2-year follow-up CBCT image with no periapical changes.

\section{DISCUSSION}

Generally taurodontic tooth there will have varied size and shape of the pulp chamber, deeper level of root canal orifice, varying degrees of canal configuration and root canal systems. ${ }^{3}$ Endodontically, taurodontism presents a challenge during different phases of the root canal procedure like canal location, the biomechanical preparation, and obturation. Since there will be a complex root canal anatomy and proximity of buccal orifices, complete filling of the root canal system in taurodont teeth is different from the normalcy. To the best of author's knowledge, this is the first case report on the taurodontic tooth treated

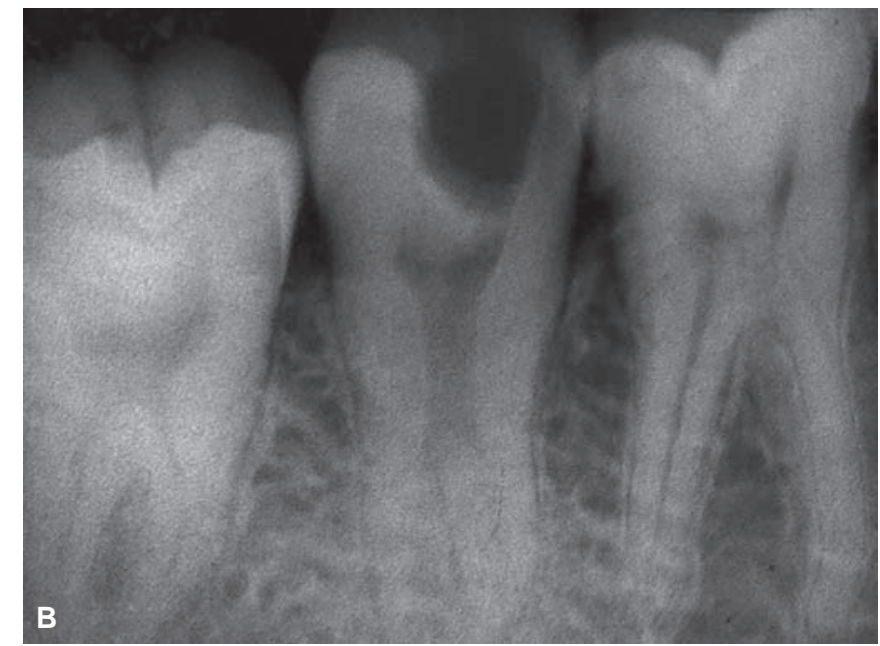

Figs 1 A and B: (A) Intraoral picture of preoperative picture of 47; (B) Preoperative radiograph of 47 exhibiting mesotaurodontism with radiolucency involving the pulp horn
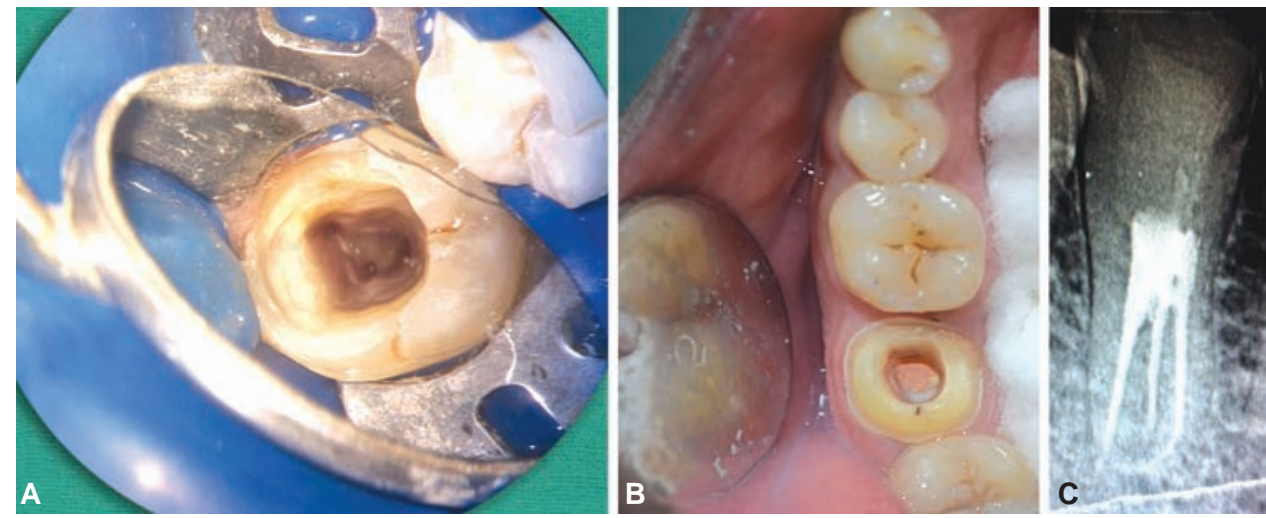

Figs 2A to C: (A) Access opening of 47 showing the canal orifices; (B) Mirror image of 47 revealing obturation; (C) Postoperative radiograph of obturated tooth 


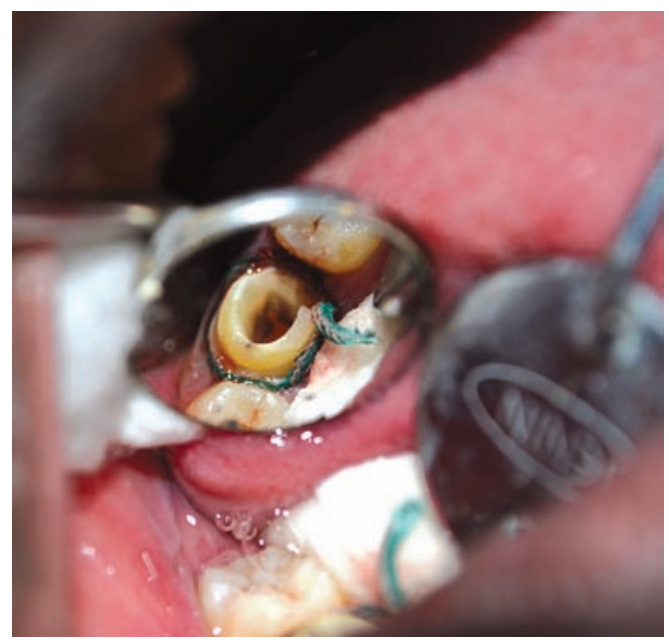

Fig 3: Placement of retraction cord

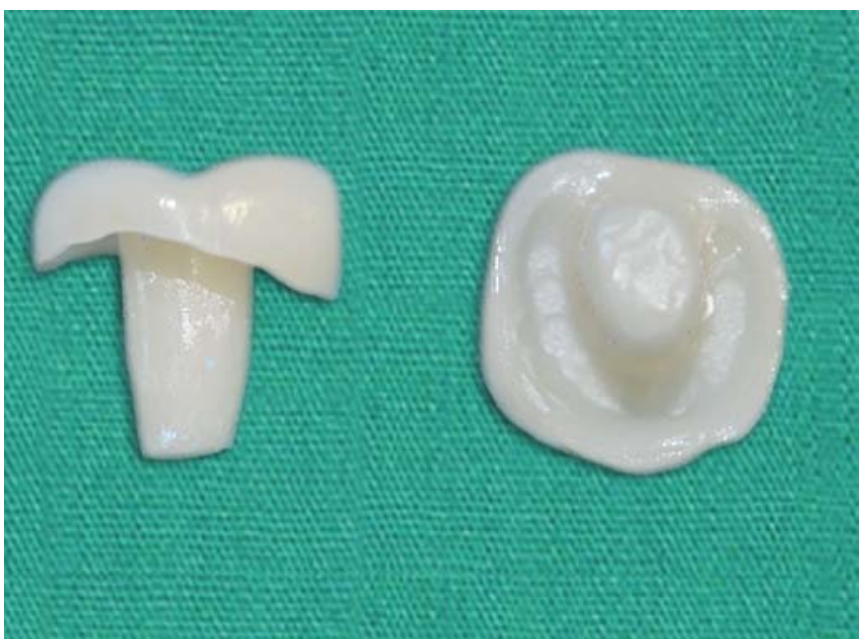

Fig 5: CAD-CAM milled crown

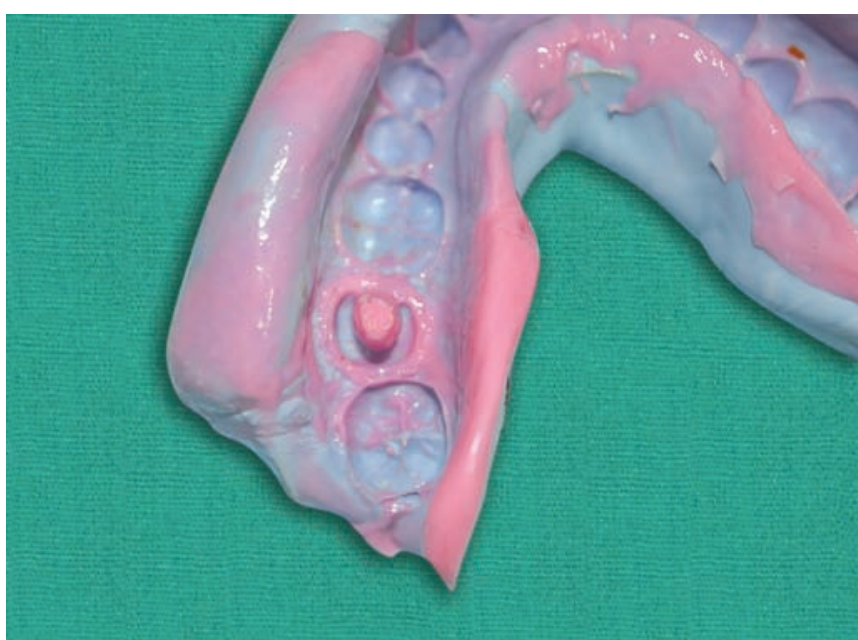

Fig 4: Two stage impression of the prepared crown along with the shape of the pulp chamber

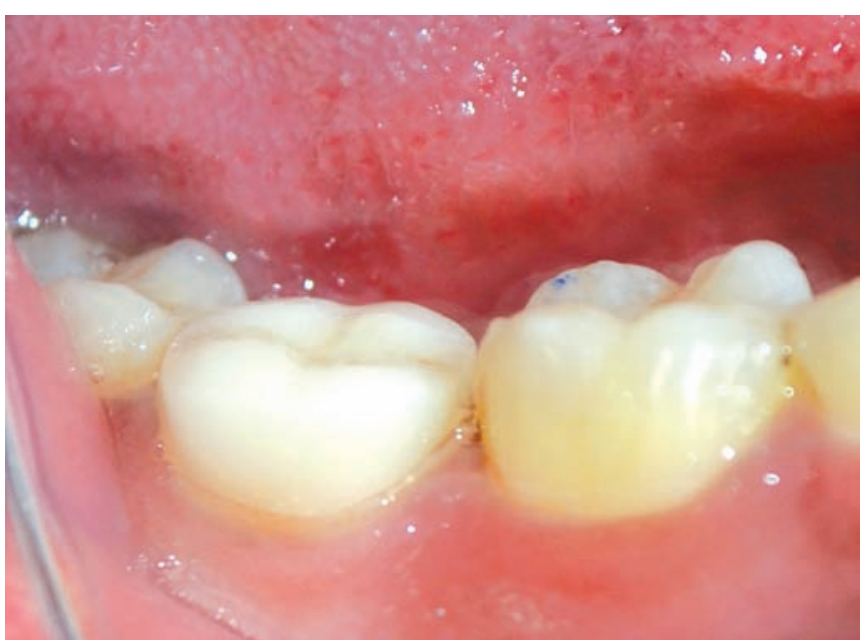

Fig 6: Postoperative picture of the cemented crown
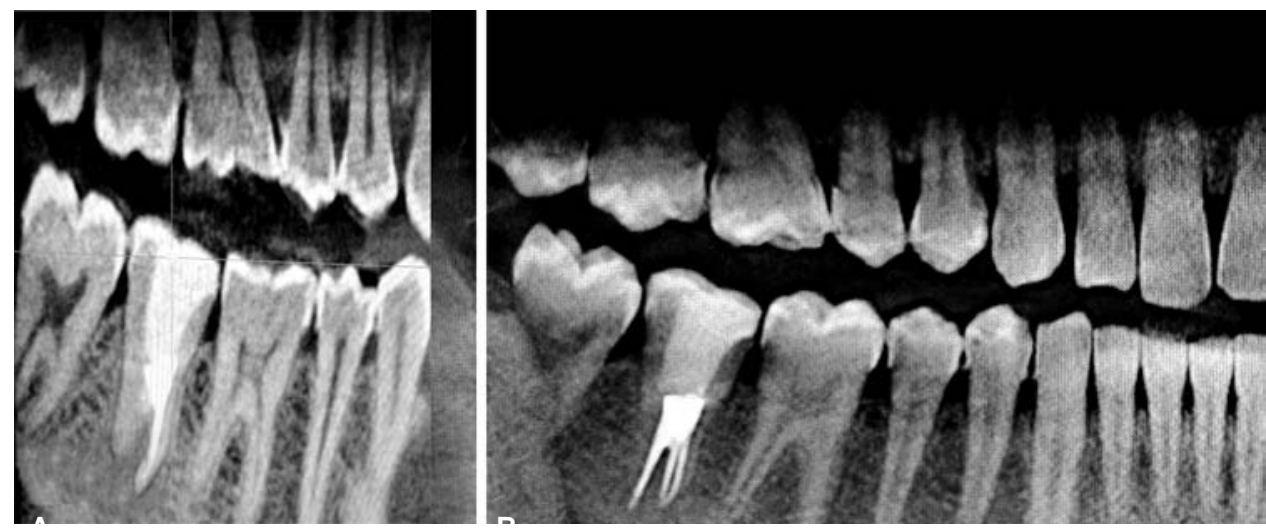

Figs 7A and B: (A) СBCT image of 47 region; (B) CBCT image of maxillary and mandibular dentition-2 year follow-up

endodontically with endocrown for post-endodontic coronal restoration. This case presented with mesotaurodont which was endodontically management with the rotary system.

After root canal treatment, restoring the tooth to reverse its oral function is a tough task especially in the grossly decayed tooth. The restoration must achieve adequate retention and evade damage to the remaining hard tissue through the prevention of bacterial microleakage and fracture of remaining tooth structure. Various treatment options are available for endodontically treated with different types of post and core systems and endocrowns. When up to one half of the coronal tooth structure is missing, complete occlusal coverage is achieved conservatively using endocrown. ${ }^{9}$ Endocrowns have several advantages over conventional crowns. It has reduced the 
number of interfaces in the restorative system, and stress concentration is less because of the reduction in the nonhomogenous material present. ${ }^{10,11}$ Endocrown involves minimal biologic width, more surfaces for bonding from the pulpal chamber and the application of resins is also better controlled. ${ }^{12}$ Endocrowns also shows satisfactory behavior in terms of esthetics, restoration stability, and tissue preservation. ${ }^{13}$

All ceramic coronal restoration is most preferred in recent times as they offer both esthetics (form) and function. The development of CAD/CAM systems and software present with various advantages in dental practice. CAD/CAM technology offers precise milling of ceramic restorations and is of the highest degree of accuracy. Time taken to prepare CAD/CAM milled crown is very minimal compared to other laborious laboratory procedures. Restorations can be produced chair-side and can be cemented in one appointment. Cross-contamination can be avoided due to impression making and laboratory procedures are condensed. There will better patient compliance and pleasing aesthetics. ${ }^{14}$ This case also reported with higher patient compliance and esthetics. However, other option like post and core or fiber reinforced composite can also be used with one more chairside visit.

Endocrown for grossly decayed tooth and especially in a taurodontic tooth is one of the best options where more retention of the post-endodontic coronal coverage is required.

\section{REFERENCES}

1. Jafarzadeh H, Azarpazhooh A, Mayhall JT. Taurodontism: a review of the condition and endodontic treatment challenges. Int Endod J 2008 May;41(5):375-388.
2. Llamas R, Jimenez-Planas A. Taurodontism in premolar. Oral Surg Oral Med Oral Pathol 1993;75:501-505.

3. Durr DP, Campos CA, Ayers CS. Clinical significance of taurodontism. J Am Dent Assoc 1980;100:378-381.

4. Hamner JE, Witkop CJ, Metro PS. Taurodontism. Report of a case. Oral Surg Oral Med Oral Pathol 1964;18:409-418.

5. Terezhalmy GT, Riley CK, Moore WS. Clinical images in oral medicine and maxillofacial radiology. Taurodontism. Quint Int 2001;32:254-255.

6. Barker BC. Taurodontism: The incidence and possible significance of the trait. Aust Dent J 1976;21:272-276.

7. Shaw JC. Taurodont teeth in South African races. J Anat 1928;62:476-498.

8. Pissis P. Fabrication of a metal-free ceramic restoration utilizing the monobloc technique. Prac Periodontics Aesthet Dent 1995;7(5):83-94.

9. Dietschi D1, Duc O, Krejci I, Sadan A. Biomechanical considerations for the restoration of endodontically treated teeth: a systematic reviewof the literature-part 2 (Evaluation of fatigue behavior, interfaces, and in vivo studies). Quint Int 2008;39(2):117-129.

10 Zarone F, Sorrentino R, Apicella D, Valentino B, Ferrari M, Aversa R, et al. Evaluation of the biomechanical behavior of maxillary central incisors restored by means of endocrowns compared to a natural tooth: a 3D static linear finite elements analysis. Dent Mater. 2006;22(11):1035-1044.

11. Lin $\mathrm{CL}$, Chang $\mathrm{YH}$, Pai CA. Evaluation of failure risks in ceramic restorations for endodontically treated premolar with MOD preparation. Dent Mater 2011;27(5):431-438.

12. Rocca G T, Serge B. Alternative treatments for the restoration of non vital teeth. Revue d'Odonto Stomatologie 2008;37: 259-272.

13. Lander E, Dietschi D. Endocrowns: a clinical report. Quint Int 2008;39(2):99-106.

14. Chang CY, Kuo JS, Lin YS, Chang YH. Fracture resistance and failure modes of CEREC endo-crowns and conventional post and core-supported CEREC crowns. J Dent Sci 2009;4(3): 110-117. 